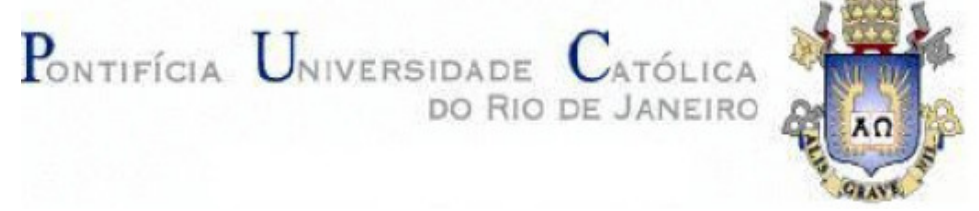

Filipe Borsato da Silva

\title{
Índice de Sentimento do Mercado Acionário no Brasil e Taxa de Retorno das Ações em Períodos Subseqüentes: Um Estudo Empírico
}

\author{
Dissertação de Mestrado
}

Dissertação apresentada ao Programa de PósGraduação em Administração de Empresas da PUC-Rio como requisito parcial para obtenção do título de Mestre em Administração de Empresas.

Orientador: Prof. Marcelo Cabus Klötzle

Rio de Janeiro

Julho de 2010 


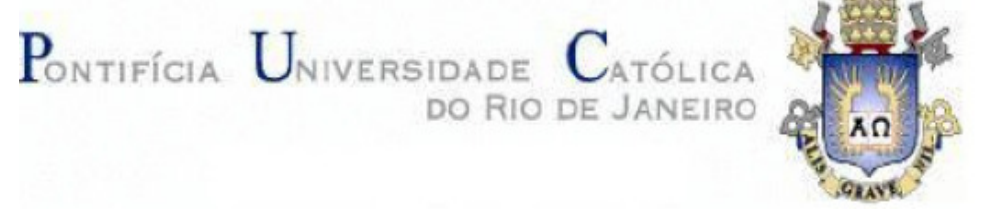

Filipe Borsato da Silva

\author{
Índice de Sentimento do Mercado Acionário no Brasil e \\ Taxa de Retorno das Ações em Períodos Subseqüentes: \\ Um Estudo Empírico
}

\begin{abstract}
Dissertação apresentada como requisito parcial para obtenção do grau de Mestre pelo Programa de Pósgraduação em Administração de Empresas da PUC-Rio. Aprovada pela Comissão Examinadora abaixo assinada.
\end{abstract}

Prof. Marcelo Cabus Klötzle Orientador Departamento de Administração - PUC-Rio

Prof. Luiz Felipe Jacques da Motta Departamento de Administração - PUC-Rio

Profa. Fernanda Finotti Cordeiro Perobelli Universidade Federal de Juiz de Fora

Prof ${ }^{a}$ Mônica Herz Vice-Decana de Pós Graduação do CCS

Rio de Janeiro, 07 de julho de 2010 
Todos os direitos reservados. É proibida a reprodução total ou parcial do trabalho sem a autorização da universidade, do autor e do orientador.

Filipe Borsato da Silva

Graduou-se em Engenharia de Telecomunicações na Universidade Federal Fluminense (UFF) em 2007.

Ficha Catalográfica

Silva, Filipe Borsato da

Índice de sentimento do mercado acionário no Brasil e taxa de retorno das ações em períodos subseqüentes: um estudo empírico / Filipe Borsato da Silva ; orientador: Marcelo Cabus Klötzle. - 2010.

68 f. : il. (color.) ; $30 \mathrm{~cm}$

Dissertação (mestrado)-Pontifícia Universidade Católica do Rio de Janeiro, Departamento de Administração, 2010.

Inclui bibliografia 
Para a minha família e para a minha amiga Cláudia 


\section{Agradecimentos}

Agradeço aos meus pais pelo carinho e apoio irrestritos e às minhas irmãs, pela compreensão e auxílio no dia a dia.

Agradeço aos meus amigos por entenderem minha ausência nos períodos em que os estudos demandaram mais de mim.

Agradeço aos colegas de mestrado por deixarem a rotina de estudos mais divertida e aos colegas de trabalho pelo apoio sempre que necessário. 


\section{Resumo}

Silva, Filipe Borsato da; Klötzle, Marcelo Cabus. Índice de Sentimento do Mercado Acionário no Brasil e Taxa de Retorno das Ações em Períodos Subseqüentes: Um Estudo Empírico. Rio de Janeiro, 2010. 68p. Dissertação de Mestrado - Departamento de Administração, Pontifícia Universidade Católica do Rio de Janeiro.

A teoria clássica de finanças considera que os investidores racionais são capazes de arbitrar ou desfazer distorções provocadas por investidores menos racionais ou providos de sentimento. Entretanto, distorções persistentes nos preços de ativos de mercado levam a crer que há limites à arbitragem. Novos modelos visam a entender como fatores comportamentais influenciam o retorno futuro das ações, de modo a explicar as flutuações de mercado e indicar a formação de bolhas especulativas. Sendo assim, o presente trabalho tem como objetivo construir um índice de sentimento do mercado brasileiro de janeiro de 2002 a dezembro de 2009, verificando as relações entre o sentimento do mercado e a precificação das ações em períodos subseqüentes. Dentre as variáveis para a construção do índice de sentimento, lança-se mão de indicadores relacionados a transações na Bolsa de Valores de São Paulo e na Comissão de Valores Mobiliários brasileira, além do índice de confiança do consumidor. Os resultados deste estudo empírico indicam que há uma relação negativa entre o sentimento dos investidores e a precificação dos ativos no trimestre subseqüente, sendo esta relação mais forte em empresas que apresentam maior risco e menor valor de mercado.

\section{Palavras-Chave}

Índice de Sentimento; Retorno Acionário; Índice de Confiança do Consumidor. 


\section{Abstract}

Silva, Filipe Borsato da; Klötzle, Marcelo Cabus (Advisor). Sentiment Index in the Brazilian Market and Stock Returns in Subsequent Periods: An Empirical Study. Rio de Janeiro, 2010. 68p. MSc. Dissertation Departamento de Administração, Pontifícia Universidade Católica do Rio de Janeiro.

The classical finance theory considers that rational investors are capable of arbitrate or offset distortions provoked by less rational investors. However, persistent distortions in asset pricing suggest that there are limits to arbitrage. New models intend to understand how behavioral factors influence the stock return in subsequent periods, in order to explain market fluctuation and the formation of speculative bubbles. The main objective of the present work is to build a sentiment index of the Brazilian market, from January 2002 until December 2009, verifying the relations between market sentiment and the value of some stocks in the subsequent period. Among the variables used in the sentiment index, there are indicators related to transactions in the São Paulo Stock Exchange and the Brazilian Securities and Exchange Commission and the consumer confidence index. The results of this empirical study indicate that there is a negative relation between the investors' sentiment and the asset pricing in the following quarter. This relation is stronger in companies that are riskier and have smaller market value.

\section{Keywords}

Sentiment Index; Stock Return; Consumer Confidence Index. 


\section{Sumário}

1. Introdução 14

1.1. Contexto do Estudo 14

1.2. Objetivos 15

1.3. Relevância 16

1.4. Delimitações do escopo do estudo 17

$\begin{array}{ll}\text { 1.5. Descrição dos Capítulos } & 17\end{array}$

2. Revisão de Literatura 18

$\begin{array}{ll}\text { 2.1. Desequilíbrios na precificação de ativos } & 18\end{array}$

2.2. Sentimento 19

2.3. Índice de Sentimento no Mercado 21

3. Metodologia 24

3.1. Proposição e Método de Pesquisa 24

3.2. Definição das Variáveis 24

3.3. Operacionalização das Variáveis 26

3.3.1. Variáveis do Índice de Sentimento 26

3.3.1.1. Número de ofertas públicas iniciais de ações 26

3.3.1.2. Percentual de ações sobre novas emissões 26

3.3.1.3. Índice de Confiança do Consumidor 27

3.3.1.4. Índice de Turnover 29

3.3.1.5. Prêmio de dividendos 30

3.3.2. Variáveis da carteira analisada 31

3.3.2.1. Segmentação da Carteira por Risco 32

3.3.2.2. Segmentação da Carteira por Valor de Mercado 32

3.3.2.3. Segmentação da Carteira por Tempo de Listagem 32

3.3.3. Limitações 33

3.4. Análise de Componentes Principais 33 
4. Análises e Resultados 36

4.1. Construção do Índice de Sentimento 36

4.1.1. Temporalidade das Variáveis 36

4.1.2. Correção de Efeitos Macroeconômicos 39

4.1.3. Definição dos Índices de Sentimento 42

4.1.3.1. Índice de Sentimento com dois Componentes Principais 43

4.2. Retorno das Ações 46

$\begin{array}{ll}\text { 4.2.1. Carteira segmentada por Risco } & 47\end{array}$

4.2.2. Carteira segmentada por Valor de Mercado 52

4.2.3. Carteira segmentada por Tempos de Listagem 55

5. Conclusões e Recomendações 60

6. Referências Bibliográficas $\quad 62$

Apêndice A. Índice de Sentimento $\quad 66$

$\begin{array}{ll}\text { Apêndice B. Carteira Analisada } & 67\end{array}$ 


\section{Lista de Tabelas}

Tabela 4.1 - Estatísticas descritivas das variáveis de sentimento

Tabela 4.2 - Correlação das variáveis de sentimento com o

índice de primeiro estágio

Tabela 4.3 - Matriz de correlações entre variáveis de sentimento

$\begin{array}{ll}\text { e RESID } & 40\end{array}$

Tabela 4.4 - Componentes Principais das variáveis de SENT2 $\perp_{t} \quad 43$

Tabela 4.5 - Variância explicada pelos componentes principais 43

Tabela 4.6 - Matriz de autovetores ou pesos da Matriz de Correlação 44

Tabela 4.7 - Matriz de autovetores ou pesos da Matriz de Correlação 44 


\section{Lista de Figuras}

Figura 4.1 - Evolução dos Índices de Sentimento do Mercado, do Índice de Sentimento do Mercado Ortogonalizado e do Índice Ibovespa

Figura 4.2 - Scree Plot de SENT $\perp_{t}$

Figura 4.3 - Evolução dos Índices de Sentimento do Mercado

Ortogonalizados com 1 e 2 Componentes Principais e evolução do Índice Ibovespa

Figura 4.4 - Média dos retornos trimestrais por risco anualizado 48

Figura 4.5 - Média dos retornos trimestrais por risco anualizado, após trimestres de $S E N T \perp_{t}$ positivo e de $S E N T \perp_{t}$ negativo Figura 4.6 - Média dos retornos trimestrais por risco anualizado, após trimestres de SENT2 $\perp_{t}$ positivo e de SENT2 $\perp_{t}$ negativo

Figura 4.7 - Média dos retornos trimestrais por risco anualizado, após trimestres de $\mathrm{SENT}^{\perp} \perp_{\mathrm{t}}$ crescente e de $\mathrm{SENT}^{\perp} \perp_{\mathrm{t}}$ decrescente

Figura 4.8 - Média dos retornos trimestrais por risco anualizado, após trimestres de SENT2 $\perp_{t}$ crescente e de SENT2 $\perp_{t}$ decrescente Figura 4.9 - Média dos retornos trimestrais por valor de mercado

Figura 4.10 - Média dos retornos trimestrais por valor de mercado, após trimestres de $\mathrm{SENT}^{\perp_{\mathrm{t}}}$ positivo e de $\mathrm{SENT} \perp_{\mathrm{t}}$ negativo

Figura 4.11 - Média dos retornos trimestrais por valor de mercado, após trimestres de $S E N T 2 \perp_{t}$ positivo e de SENT2 $\perp_{t}$ negativo

Figura 4.12 - Média dos retornos trimestrais por valor de mercado, após trimestres de $\mathrm{SENT}^{\perp} \perp_{\mathrm{t}}$ crescente e de $\mathrm{SENT} \perp_{\mathrm{t}}$ decrescente

Figura 4.13 - Média dos retornos trimestrais por valor de mercado, após trimestres de SENT2 $\perp_{t}$ crescente e de SENT2 $\perp_{t}$ decrescente

Figura 4.14 - Média dos retornos trimestrais por tempo de listagem na Bovespa 
Figura 4.15 - Média dos retornos trimestrais por tempo de listagem, após trimestres de $\mathrm{SENT}^{\perp}{ }_{\mathrm{t}}$ positivo e de $\mathrm{SENT}^{\perp}{ }_{\mathrm{t}}$ negativo

Figura 4.16 - Média dos retornos trimestrais por tempo de listagem, após trimestres de SENT2 $\perp_{t}$ positivo e de SENT2 $\perp_{t}$ negativo

Figura 4.17 - Média dos retornos trimestrais por tempo de listagem, após trimestres de $\mathrm{SENT}^{\perp_{\mathrm{t}}}$ crescente e de $\mathrm{SENT}^{\perp_{\mathrm{t}}}$ decrescente

Figura 4.18 - Média dos retornos trimestrais por tempo de listagem, após trimestres de SENT2 $\perp_{t}$ crescente e de SENT2 $\perp_{t}$ decrescente 


\section{Lista de Acrônimos}

BOVESPA

CAPM

CCI

CI

$\mathrm{CNI}$

COPOM

CVM

DIV

FECOMERCIO-RJ

FECOMERCIO-SP

FGV

IBRX

IBOVESPA

ICC

ICEA

IE

IEC

INEC

IPO

NIPO

S

SENT

TURN
Bolsa de Valores de São Paulo

Capital Asset Pricing Model

Consumer Confidence Index Index of Current Economic Expectations Confederação Nacional da Indústria Comitê de Política Monetária do Banco Central Comissão de Valores Mobiliários

Prêmio de Dividendos

Federação do Comércio do Estado do Rio de Janeiro Federação do Comércio do Estado de São Paulo Fundação Getúlio Vargas Índice Brasil Índice BOVESPA Índice de Confiança do Consumidor Índice de Condições Econômicas Atuais Index of Consumer Expectations Índice de Expectativas do Consumidor Índice Nacional de Expectativas do Consumidor Initial Public Offering Número de IPOs Proporção de Emissão de Ações Frente Emissão total de Debêntures e Notas Promissórias Índice de Sentimento do Mercado Turnover de Ações 